\title{
Utilisation de la diffraction de rayons $X$ pour l'étude de traitements de surface laser
}

\author{
F. Hlawka, G.Q. Song et A. Cornet \\ E.N.S.A.I.S., L.M.C.M., 24 boulevard de la Victoire, 67084 Strasbourg, France
}

\begin{abstract}
Résumé : Le traitement de surface par refusion laser et injection de particules dures permet de créer en surface des alliages d'une résistance à l'usure remarquable. Pour éviter certains défauts importants, tels que porosité et fissuration, la détermination des phases et l'étude de la nature des contraintes résiduelles apparaissant en surface sont primordiales. La diffraction de rayons $X$ permet donc dans ce domaine de guider le choix des paramètres pour optimiser le résultat du traitement. Dans notre cas, nous avons pu ainsi réaliser à partir d'un simple acier de type $35 C D 4$ un alliage ayant des performances proches de celles des aciers à outils beaucoup plus chers, grâce à l'injection de carbure de bore et de zircone.
\end{abstract}

\begin{abstract}
Laser surface treatments with remelting and hard particles injection induce the creation of wear resisting alloys. To prevent important defects like porosity or cracking, the determination and the distribution of the metallurgical phases and the study of the residual stresses are fundamental. That's the reason why $X$-ray diffraction is an appropriate method to guide the choice of laser treatment parameters in order to optimize the alloying result. In that work an ordinary structural AISI $4135 \mathrm{H}$ steel has been used and superficially laser alloyed with the injection of both boron carbide and zircone: it presents a great hardness, no cracking and could serve as a substitute for expensive cutting tool steels.
\end{abstract}

\section{INTRODUCTION}

\subsection{But du traitement laser}

La réalisation d'alliages de surface par injection de poudre de particules dures sous faisceau laser permet de modifier localement des pièces pour lesquelles sont requises en surface certaines propriétés caractéristiques telles que résistance à la corrosion, à l'usure [1] ... Les essais ont été menés ici sur des aciers susceptibles d'être utilisés en tant qu'aciers à outils.

\subsection{Utilisation de la diffraction de rayons $X$}

La nature des phases métalliques qui apparaissent lors de ces traitements est difficilement prévisible à partir des diagrammes d'équilibre [2], le refroidissement suivant la refusion laser s'établissant à une vitesse beaucoup trop élevée même par rapport aux phénomènes de trempe classique.

Moyennant un tronçonnage préalable du cordon laser en coupes parallèles, par la suite usinées et polies, la diffraction de rayons $X$ permet de découvrir les différentes phases depuis la surface jusqu'au fond du cordon. De plus elle permet de déterminer la nature des contraintes résiduelles après traitement qui jouent un rôle considérable dans le comportement mécanique de ces nouvelles couches dont les défauts majeurs sont le plus souvent une tendance à la fissuration et une porosité excessive.

\section{CONDITIONS EXPERIMENTALES}

\subsection{Dispositif laser}

L'échantillon d'acier à traiter se déplace à vitesse constante $\mathrm{Vb}$ comprise entre 0,5 et 10 $\mathrm{m} / \mathrm{min}$ devant le faisceau d'un laser $\mathrm{CO}_{2}$ de $4 \mathrm{~kW}$ de puissance, de $4 \mathrm{~mm}$ de diamètre de défocalisation muni d'un système d'apport de poudre avec protection de gaz coaxiale (Fig1). Les valeurs du débit de poudre de carbure de bore varient entre $0,7 \mathrm{~g} / \mathrm{min}$ et $2,4 \mathrm{~g} / \mathrm{min}$. 
Le cordon obtenu comporte plusieurs zones où la répartition et la nature des phases sont déterminantes dans la tenue à l'abrasion de cet alliage (Fig.2).

\subsection{Diffraction de rayons $X$}

\subsubsection{Détermination des phases}

Après tronçonnage, usinage et polissage de chaque couche (Fig.3) les échantillons ont été étudiés à l'aide d'un diffractomètre SIEMENS D5000, muni d'une anode de cobalt (ou de chrome) avec un filtre de fer (ou vanadium), ce qui permet l'exploration des phases depuis la surface jusqu'au fond du cordon (Fig.4).

\subsubsection{Etude des contraintes résiduelles}

L'état de contrainte a été testé dans le sens longitudinal et transversal des cordons sur les plan $\{211\}$ du fer avec un tube de rayons X équipé d'une anode en chrome et obtenu en utilisant la méthode d'exposition multiple [3]; le tracé des droites $\mathrm{d}=\mathrm{f}\left(\sin ^{2} \Psi\right)$ permet alors de connaître le signe des macrocontraintes.

\section{RESULTATS}

Les outils de coupe, très sollicités dans leur utilisation, sont généralement réalisés en acier rapide. Nous avons tout d'abord voulu améliorer par traitement laser les performances de l'acier M42 en lui injectant du carbure de bore, une particule très dure, puis nous avons testé le même type de traitement sur un acier de construction générale, le 35CD4.

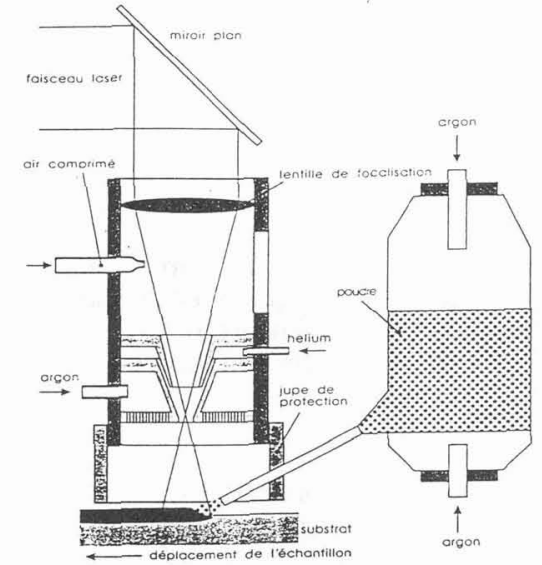

Fig.1 : Schéma du dispositif utilisé pour la refusion avec injection de poudre

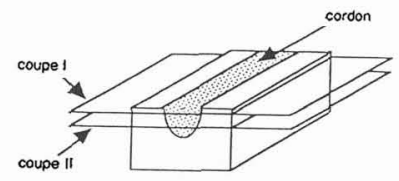

Fig.3 : Schématisation de la coupe d'un échantillon traité laser dans le but de l'analyse par diffraction $X$

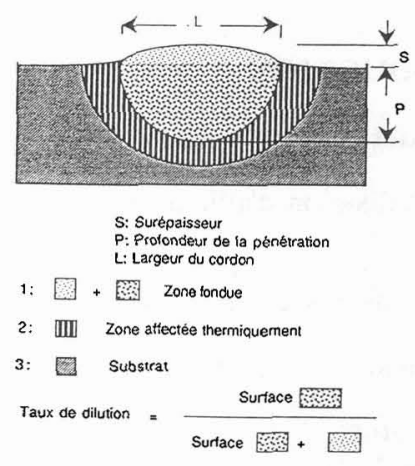

Fig.2 : Géométrie du cordon

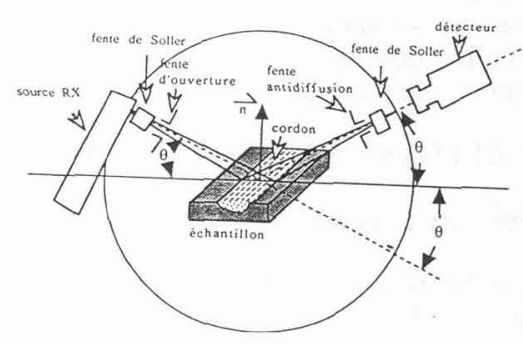

Fig.4 : Dispositif d'étude du cordon laser par diffraction de rayons $\mathrm{X}$ 


\subsection{Injection de $\mathrm{B}_{4} \mathrm{C}$ dans l'acier rapide $\mathrm{M} 42$}

Une combinaison de paramètres optimisée (puissance laser, vitesse de balayage, débit de poudre, couverture gazeuse) donne un cordon non poreux et non fissuré, bien adhérent au substrat.

La zone fondue présente des valeurs de dureté comprises entre $1300 \mathrm{HV}$ et $1900 \mathrm{HV}$ (Fig.5) due à la présence de borures de fer $\mathrm{Fe}_{3} \mathrm{~B}, \mathrm{Fe}_{23} \mathrm{~B}_{6}$ et de carbure de bore non dissous.

\subsection{Injection de $\mathrm{B}_{4} \mathrm{C}$ dans l'acier $35 \mathrm{CD} 4$}

Les diverses combinaisons de paramètres aboutissent toujours à une fissuration : seul un préchauffage préalable de la pièce à $580^{\circ} \mathrm{C}$ permet de l'éviter [4]. Dans ce cas, la dureté obtenue prend des valeurs comprises entre $1400 \mathrm{HV}$ et $1800 \mathrm{HV}$ (Fig.6) due à la formation de la phase $\mathrm{Fe}_{2} \mathrm{~B}$ (Fig.7).

\subsection{Injection d'un mélange de $\mathrm{B}_{4} \mathrm{C}$ et de zircone dans l'acier 35CD4}

Dans le but de simplifier le traitement en supprimant le préchauffage, nous avons ajouté de la zircone au carbure de bore (des mélanges de $10 \%$ à $30 \%$ ont été testés): les particules de zircone changent de volume au cours du traitement et induisent des contraintes résiduelles en compression (Fig.8), tout en n'altérant pas la phase $\mathrm{Fe}_{2} \mathrm{~B}$ toujours présente dans l'alliage. On obtient un cordon bien homogène, bien adhérent au substrat et présentant une dureté de l'ordre de 1400 HV (Fig.9).

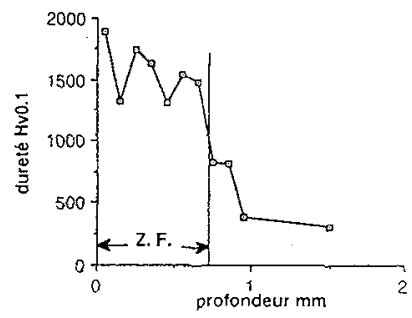

Fig.5: Evolution de la microdureté en fonction de la profondeur (Acier rapide $\mathrm{M} 42$ allié $\mathrm{B}_{4} \mathrm{C}$ par faisceau laser)

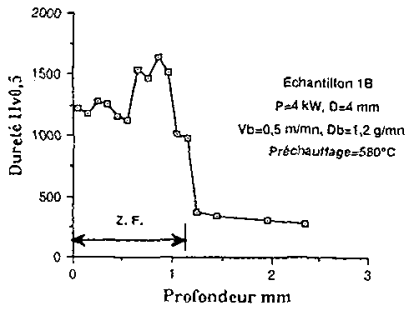

Fig.6 : Evolution de la microdureté en fonction de la profondeur (Acier $35 \mathrm{CD} 4$ allié $\mathrm{B}_{4} \mathrm{C}$ par faisceau laser)

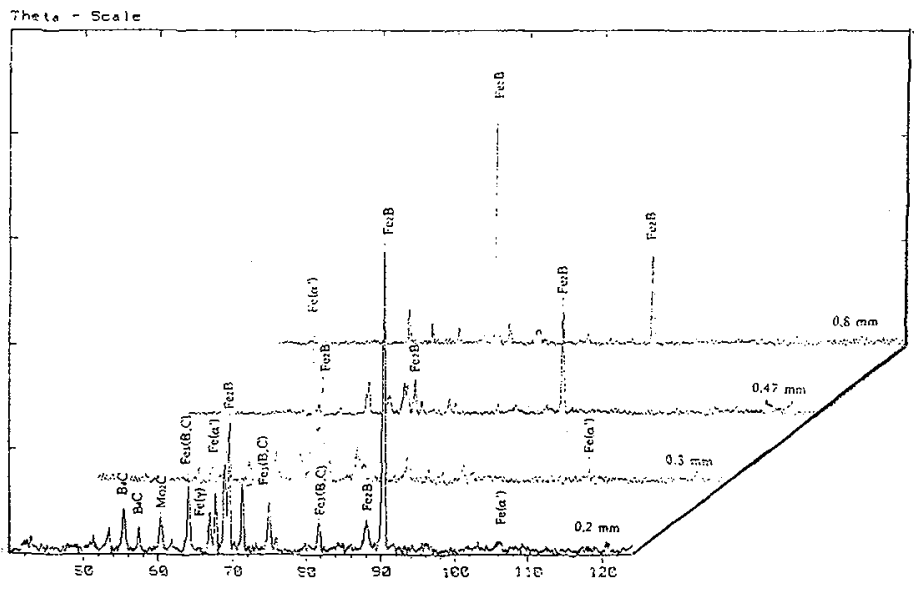

Fig.7 : Diagrammes de diffraction $\mathrm{X}$ suivant différentes profondeurs dans la zone fondue Tube à rayons $\mathrm{X}: \mathrm{Cr}$ 


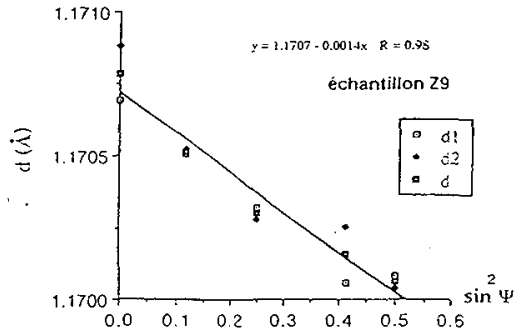

Fig.8: Evolution de la distance interréticulaire de plan cristallin en fonction de $\sin ^{2} \Psi$ pour $35 \mathrm{CD} 4$ allié $\quad \mathrm{B}_{4} \mathrm{C}+\mathrm{ZrO}_{2}$ par laser (sens longitudinal du cordon)

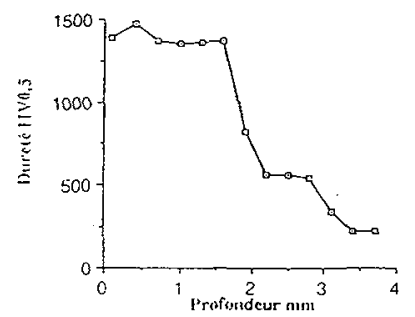

Fig.9 : Evolution de la microdureté en fonction de la profondeur (Acier $35 \mathrm{CD} 4$ allié $\mathrm{B}_{4} \mathrm{C}+\mathrm{ZrO}_{2}$ par faisceau laser)

\section{CONCLUSION}

Tout au long de cette étude, la diffraction de rayons $\mathrm{X}$ nous a quidés, tant dans la détermination des phases présentes dans le cordon laser que dans l'étude du signe des contraintes résiduelles à sa surface.

Elle nous a permis d'établir le rôle stabilisant de la zircone au niveau de la répartition des contraintes occasionnées par l'introduction de particules dures dans un substrat sous faisceau laser et de réaliser à partir d'un acier ordinaire un alliage de surface digne de rivaliser avec les aciers rapides.

\section{REFERENCES}

[1] HLAWKA F. et al, Surface Engineering vol.9, No.4, (1993) 300 - 304.

[2] ERBNER R., Proc. 2nd European Conf. on Advanced Materials and Processes, Cambrige (1991) S. 115.

[3] CASTEX L. et al, Détermination des contraintes résiduelles par diffraction des rayons $X$ (l'ENSAM, Paris, 1981) pp.77 - 80.

[4] SONG G.Q., HLAWKA F., CORNET A., Lasers de Puissance, 10e Colloque Université industrie, 15 juin (1995) C81-C86. 